\title{
Spontaneous Oscillations of a Minimal Actomyosin System under Elastic Loading
}

\author{
P.-Y. Plaçais, M. Balland, T. Guérin, J.-F. Joanny, and P. Martin* \\ Laboratoire Physico-Chimie Curie, CNRS, Institut Curie, UPMC; 26 rue d'Ulm, F-75248 Paris Cedex 05, France
}

(Received 23 June 2009; published 9 October 2009)

\begin{abstract}
Spontaneous mechanical oscillations occur in various types of biological systems where groups of motor molecules are elastically coupled to their environment. By using an optical trap to oppose the gliding motion of a single bead-tailed actin filament over a substrate densely coated with myosin motors, we mimicked this condition in vitro. We show that this minimal actomyosin system can oscillate spontaneously. Our finding accords quantitatively with a general theoretical framework where oscillatory instabilities emerge generically from the collective dynamics of molecular motors under load.
\end{abstract}

Mechanical oscillations occur in a variety of biological systems. Insect fibrillar flight muscles develop oscillatory tension with a rhythm that is asynchronous to activating nervous impulses [1]. Skinned skeletal and cardiac muscle fibers also exhibit spontneous oscillations in vitro under various conditions of partial activation [2,3], for the latter even in the absence of proteins that normally regulate contractility of the muscle [4]. Spontaneous oscillations have also been observed in nonmuscular motor systems. Oscillations of the mitotic spindle during asymmetric cell division [5], of some insects' antennal hearing organs [6], and of mechanosensory hair bundles in hair cells from the vertebrate ear [7] offer illustrative examples. In the last case, mechanical oscillations have been shown to provide amplification of weak stimuli and frequency tuning [8].

All these active mechanical systems rely on a forceproducing machinery that involves molecular motors working in groups. To generate oscillations, these motors must work against an elastic load. Muscle fibers, for instance, oscillate in vitro only when attached at both ends [3]. Here we have modified a conventional gliding assay to test the ability of a minimal actomyosin system to produce spontaneous oscillations under elastic loading. A single actin filament was attached to a micron-sized silica bead, either by mixing biotinylated actin filaments with streptavidin-coated beads or by using beads functionalized with inactivated myosins [9] or gelsolin [10]. All three methods yielded similar results. The bead served both as a handle to manipulate the filament with optical tweezers and as a reporter of the position of the filament end to which it was bound. Such a filament could be captured in bulk and brought in close proximity to a substrate densely coated with heavy meromyosin molecules from rabbit skeletal muscle (Fig. 1).

In the presence of $2 \mathrm{mM}$ ATP, position fluctuations of the bead within the optical trap displayed three qualitative changes upon interaction of the actin filament with myosin molecules [Fig. 2(a)]. First, the average position of the bead moved away from the center of the optical trap, indicating that the actin filament was set under tension.
The baseline of bead position shifted in the positive direction by $6-64 \mathrm{~nm}$ ( $n=11$ measurements). Accordingly, the trap exerted a mean restoring force of $2-17 \mathrm{pN}$ that opposed action of the motors. Second, the bead displayed a protracted motion along a well-defined direction. The rootmean-squared (rms) magnitude along this axis increased by a factor $4.0 \pm 1.6$ (mean $\pm \mathrm{SD}, n=29$ measurements) with respect to that of the isotropic Brownian motion of the free bead. For trap stiffnesses $k_{T}=0.05-0.39 \mathrm{pN} \cdot \mathrm{nm}^{-1}$, motor-driven movements displayed rms magnitudes of 3.3-29.7 nm, corresponding to peak-to-peak magnitudes of 9-87 nm. Finally, a striking feature of our recordings was the presence of noisy spontaneous oscillations. Oscillations persisted for about 25-65 cycles. Rhythmic activity was characterized by the presence of a clear peak in the spectral density of bead position [Figs. 2(b) and 2(c)]. The peak was centered at a frequency $\nu_{0}=$ $1.5-14 \mathrm{~Hz}(n=29)$, which defined the characteristic frequency of oscillation. Frequency fluctuations around $\nu_{0}$ could be described by the quality factor $Q=\nu_{0} / \Delta \nu=$ $1.4 \pm 1.1$ (mean $\pm \mathrm{SD}$ ), where $\Delta \nu$ corresponds to the width of the spectral density at half its maximal value.

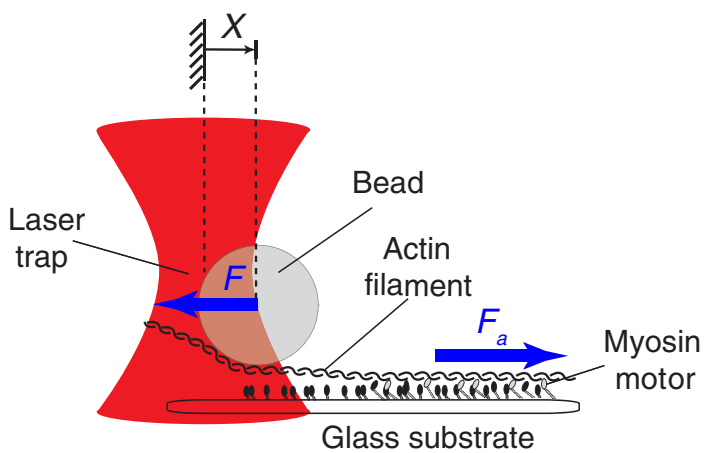

FIG. 1 (color online). Gliding assay with elastic loading. The motor molecules develop an active force $F_{a}$ on the actin filament in a direction defined here as positive. The optical trap imposes an elastic restoring force $F=-k_{T} X$ in the negative direction. $k_{T}$ represents the trap stiffness and $X$ the position of the bead with respect to the center of the trap. 
(a)

(b)
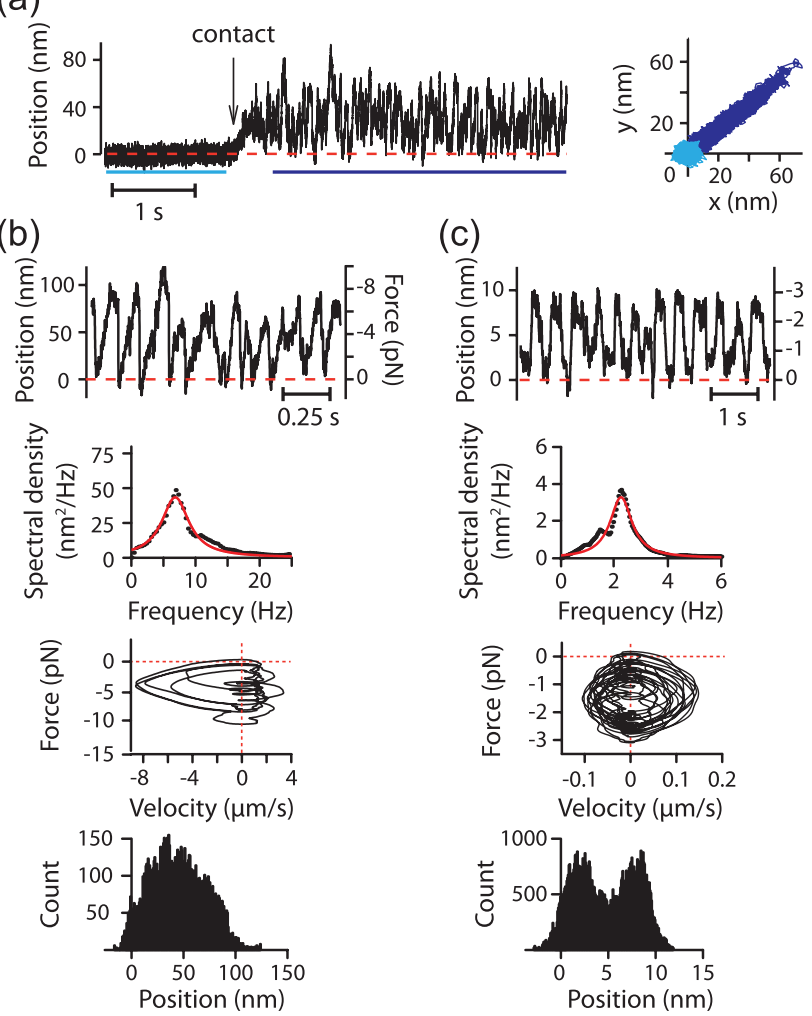

(c)
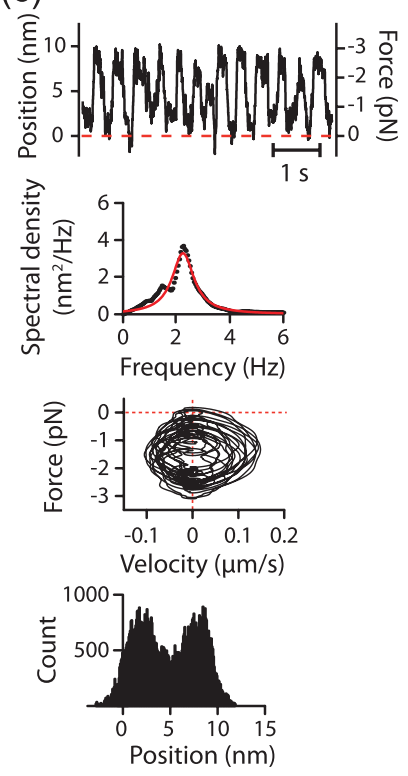

FIG. 2 (color online). (a) Time course of bead position at the onset of interaction between actin and myosin (left) and bead trajectories in the horizontal plane before and after contact (right). In (b) and (c), from top to bottom, we show spontaneous oscillations of bead position (with corresponding trap forces), their spectral densities (smoothed and fitted with a Lorentzian function), relations between trap force and bead velocity, and position histograms. (b) Mean position, $+50 \mathrm{~nm}$; rms magnitude, $29 \mathrm{~nm}$; peak-to-peak magnitude, $\sim 80 \mathrm{~nm}$; spectrum peak at $\nu_{0}=6.9 \mathrm{~Hz}$; quality factor $Q=1.3$. The trap stiffness was here $k_{T}=0.083 \mathrm{pN} \cdot \mathrm{nm}^{-1}$. (c) Mean position, $4.8 \mathrm{~nm}$; rms magnitude, $3.2 \mathrm{~nm}$; peak-to-peak magnitude, $\sim 8.5 \mathrm{~nm}$; spectrum peak at $\nu_{0}=2.2 \mathrm{~Hz}$; quality factor was $Q=2.3$. Here, $k_{T}=0.31 \mathrm{pN} \cdot \mathrm{nm}^{-1}$.

Correspondingly, a bead oscillation lost its phase coherence after only a few cycles. Within the most regular oscillations $(Q \geq 1)$, it was sometimes possible to recognize a sawtooth motif in which a slow excursion at a nearly constant velocity, $\sim 1.1 \mu \mathrm{m} \cdot \mathrm{s}^{-1}$ in the example shown in Fig. 2(b), was followed by a rapid stroke in the opposite direction. Notably, the slow component of motion was always in the positive direction (defined in Fig. 1). In this case, the probability distribution of bead position remained maximal within a broad region centered at the mean position. The waveform of such an asymmetric triangular oscillation resembled that of spontaneous oscillatory contractions of cardiac or skeletal muscle fibers [2]. Alternatively, we also observed approximately rectangular patterns, in which abrupt movements in both directions were interrupted by noisy pauses [Fig. 2(c)]. The position histogram was thus bimodal, with a local minimum at the mean position. These oscillations were strikingly similar to spontaneous hair-bundle oscillations measured in vitro with hair cells from the inner ear of the bullfrog [7,11]. In these two cases, the bead movement comprised components on two time scales that are typical of relaxation oscillations. Most often, however, fluctuations clouded the oscillation waveform.

The maximal absolute force $F_{\max }$ that the trap exerted during an oscillation afforded an approximate means to estimate the number of active motor proteins that produced an oscillation. Throughout 11 experiments, $F_{\max }$ varied by almost 1 order of magnitude, from 3.5 to $27.5 \mathrm{pN}$. Notably, tension in a single thin filament from an oscillatory myofibril falls within a similar range [12]. Assuming that each myosin molecule developed a force of $1.5 \mathrm{pN}$ and spent $14 \%$ of its ATPase cycle bound to actin [13], each actin filament would have recruited a pool of 15-130 myosin molecules among which 2-18 interacted with the filament at any given time. We could not control the length of the actin-filament portion available to myosins, which spanned a range of $\sim 0.5$ to $5 \mu \mathrm{m}$. This source of variability in part explained the dispersion in the number of motors that were at work for different oscillations. Considering the inherent variability in $F_{\text {max }}$ throughout our recordings, it may be no surprise that we observed no correlation between magnitude and frequency of oscillations (data not shown). However, for the few actomyosin systems that produced similar values of $F_{\max }$, we observed that a higher trap stiffness resulted in smaller and faster oscillations (Table I). This suggests that the trap stiffness is a parameter that can control, at fixed $F_{\max }$, the magnitude and frequency of oscillation.

Several mechanisms have been proposed to explain the production of spontaneous oscillations by complex motor systems. In hair cells, spontaneous hair-bundle oscillations have been interpreted as an interplay between the activity of a myosin-based adaptation motor, negative hair-bundle stiffness and $\mathrm{Ca}^{2+}$ feedback on the motor force [7]. Although negative stiffness might arise from mechanosensitivity of individual actomyosin crossbridges [14,15], there is no $\mathrm{Ca}^{2+}$ feedback in our actomyosin system. In insect flight muscle, proposed mechanisms of oscillations rely on inertial loading of the contractile apparatus and

TABLE I. Peak-to-peak magnitude $A$ and frequency $\nu_{0}$ of oscillations at a fixed maximal trap force $F_{\max }$ and variable trap stiffness $k_{T}$.

\begin{tabular}{cccc}
\hline \hline$F_{\max }(\mathrm{pN})$ & $k_{T}\left(\mathrm{pN} \cdot \mathrm{nm}^{-1}\right)$ & $A(\mathrm{~nm})$ & $\nu_{0}(\mathrm{~Hz})$ \\
\hline 25 & 0.26 & 76 & 4.3 \\
& 0.37 & 48 & 10 \\
\hline \multirow{2}{*}{8} & 0.08 & 87 & 7 \\
& 0.15 & 39.8 & 11 \\
\hline \hline
\end{tabular}


delayed stretch activation, which can compensate frictional forces and thus destabilize this spring-mass system [16]. In our experiments, however, true inertial forces can be neglected. In addition, oscillations involve only filamentous actin and a few tens of myosin molecules, without any of the regulatory proteins that are normally present in complex systems. Despite its apparent simplicity, our system comprises molecular motors which do not work in isolation but within a group. Active force production by one motor is thus potentially influenced by the behavior of all the others. Collective effects, including oscillations, may arise for instance if motor molecules displayed load-dependent detachment rates [5,14] or if geometrical constraints resulted in a modulation of the available pool of myosins to actin $[2,17]$. Oscillatory instabilities may also emerge generically from the dynamical properties of motor assemblies [18]. Because the last approach does not depend on the system's molecular details and geometry, we discuss our observations within this general theoretical framework.

In this description, the center of mass of each motor is rigidly connected to a common substrate, the motors are randomly distributed, and the actomyosin system is simply described by two states [Fig. 3(a)]. In the attached state, the interaction between actin and myosin is described by an asymmetric potential $W_{1}(\xi)$ which varies with the position $\xi$ along the actin track and displays a periodicity $l$ on the order of the actin-monomer size. Without loss of generality, we can assume that the detached state is characterized by a flat potential $W_{2}$. The actin filament glides at velocity $v=d X / d t$, and the probability density of the motors in the attached state at time $t$ is $p_{1}(\xi, t)$. Force balance between the driving force $F_{a}=\int_{0}^{l} N W_{1}^{\prime}(\xi) p_{1}(\xi, t) d \xi$ produced by the motors, an elastic restoring force given by a spring of stiffness $k_{T}$, and a frictional force characterized by a coefficient $\lambda$ yields

$$
\lambda v=-k_{T} X+F_{a}+\eta(t) .
$$

The noise term $\eta$ accounts for the stochastic nature of motor binding and unbinding to actin and is zero on average. We used uncorrelated white noise with $\langle\eta(t) \eta(0)\rangle=J \delta(t)$. A rough estimate of noise intensity $J \simeq 5 \times 10^{-27} \mathrm{~N}^{2} \mathrm{~s}$ is given by $J \simeq N f^{2} r(1-r) \tau_{m}$ [19], in which $N \simeq 50$ is the total number of motors, $f \simeq 1 \mathrm{pN}$ is the single-motor force, $\tau_{m} \simeq 1 \mathrm{~ms}$ is the characteristic time scale of force production by a motor, and $r \simeq 0.1$ is the fraction of bound motors. The Fokker-Planck equation for $p_{1}$ reads

$$
\partial_{t} p_{1}-v \partial_{\xi} p_{1}=-\omega_{\text {off }}(\xi) p_{1}+\omega_{\text {on }}(\xi)\left[1 / l-p_{1}(\xi)\right]
$$

in which $\omega_{\text {on }}(\xi)$ and $\omega_{\text {off }}(\xi)$ are the attachment and detachment rates at position $\xi$, respectively. We assumed, for simplicity, a uniform attachment rate $\omega_{\text {on }}$ and that detachment occurred only within a narrow region near the minimum of the potential $W_{1}$ in which $\omega_{\text {off }}=\Omega \gg \omega_{\text {on }}$ (a)

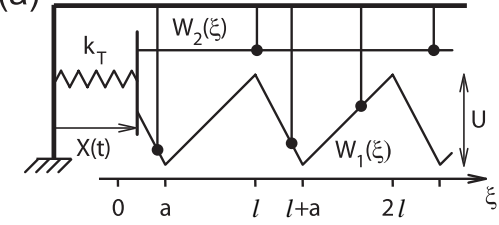

(b)

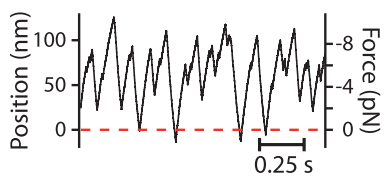

(c)
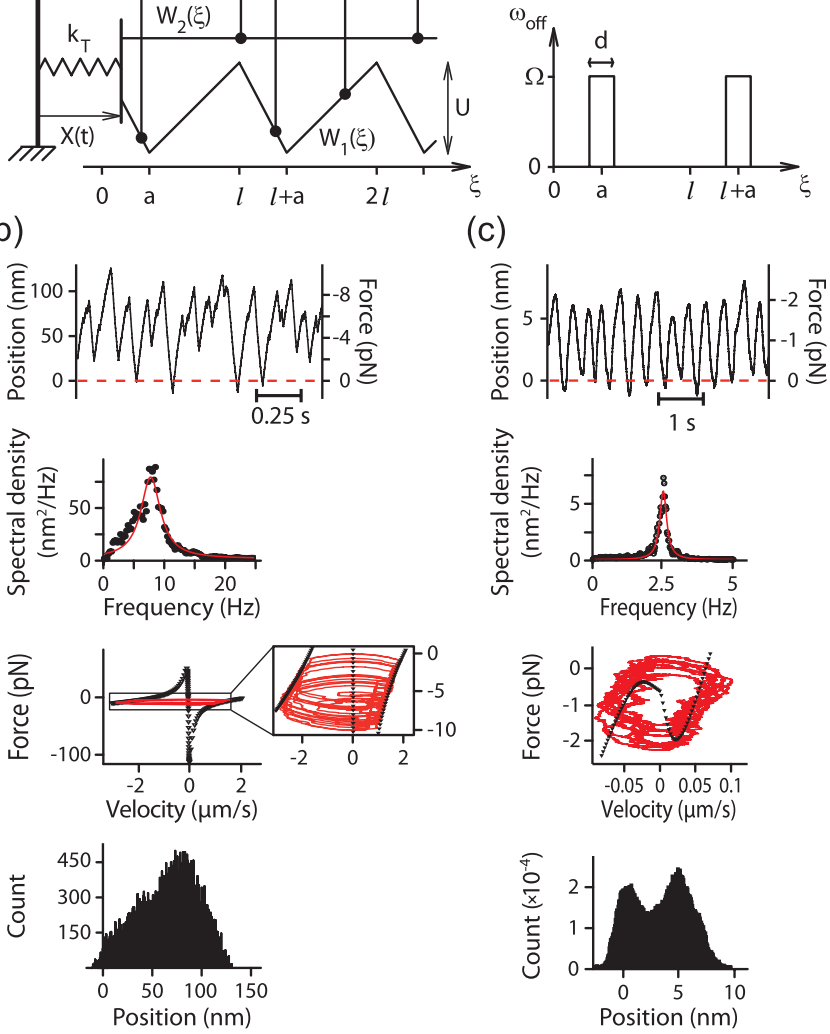

FIG. 3 (color online). Stochastic simulations. (a) Periodic potentials $W_{1}(\xi)$ and $W_{2}(\xi)$ and detachment rate $\omega_{\text {off }}(\xi)$. In (b) and (c), from top to bottom, we show spontaneous oscillations of track position, their spectral densities, the steady-state relations between the external force $\lambda v-F_{a}$ and the relative velocity $v$ between the track and the motors (black triangles) together with the system's trajectory in this plane (red lines), and position histograms. (b) Frequency, $\nu_{0}=7.2 \mathrm{~Hz}$; rms magnitude, $30.4 \mathrm{~nm}$; quality factor, $Q=1.98$. Parameter values: $l=$ $6 \mathrm{~nm}, \omega_{\text {on }}^{-1}=40 \mathrm{~ms}, a / l=0.3, d / l=0.1, \Omega / \omega_{\text {on }}=200, U=$ $4 \mathrm{pN} \cdot \mathrm{nm}, N=332, J=3.37 \times 10^{-27} \mathrm{~N}^{2} \mathrm{~s}, \lambda=3.69 \mu \mathrm{N} \cdot \mathrm{s} \cdot$ $\mathrm{m}^{-1}$, and $k_{T}=0.083 \mathrm{pN} \cdot \mathrm{nm}^{-1}$. (c) Frequency, $\nu_{0}=2.5 \mathrm{~Hz}$; rms magnitude, $2.47 \mathrm{~nm}$; quality factor, $Q=8.5$. Same parameters as in (b), but for $N=10, J=4.83 \times 10^{-27} \mathrm{~N}^{2} \cdot \mathrm{s}, \lambda=$ $49.6 \mu \mathrm{N} \cdot \mathrm{s} \cdot \mathrm{m}^{-1}$, and $k_{T}=0.31 \mathrm{pN} \cdot \mathrm{nm}^{-1}$.

[Fig. 3(a)]. This choice ensures that the detailed-balance condition is broken. Energy is thus provided to the system, here by ATP hydrolysis. In the absence of noise, the motor force (at steady state) near stall force $F_{\text {stall }}$ amounts to negative friction: $F_{a}=F_{\text {stall }}+\lambda_{a} v$ with $\lambda_{a}=$ $N U /\left[2 a(l-a) \omega_{\text {on }}\right]$. This implies that if a number of motors drive the movement of the filament in one direction, they enhance the likelihood that the others join in and pull in the same direction. If $\lambda_{a}$ overcomes the passive friction coefficient $\lambda$, the force-velocity relation of the whole system displays an anomalous region of negative slope. In the presence of an elastic element, the system in turn oscillates spontaneously [18]. For small values of the stiffness $k_{T}$, a Hopf bifurcation occurs at a critical value 
$\lambda_{a}^{*}=\lambda+3 k_{T} /\left(4 \omega_{\text {on }}\right)$ of $\lambda_{a}$, with a frequency $\nu^{*}=$ $\sqrt{3 k_{T} \omega_{\text {on }} /\left(8 \pi^{2} \lambda\right)}$.

We performed stochastic simulations of Eq. (1) in which, at each time step, the active motor force $F_{a}$ was calculated by numerical integration of Eq. (2). Very far from the bifurcation, we observed triangular oscillations of large magnitude compared to the actin-monomer size $l \sim$ $5.5 \mathrm{~nm}$ [Fig. 3(b)]. The waveform, magnitude and frequency of oscillation are in quantitative agreement with some experiments [Fig. 2(b)]. During an oscillation, the system switched stochastically between the two stable branches of its force-velocity relation, resulting in a velocity histogram that was bimodal (not shown). In the example shown in Fig. 3(b), the histogram peaked at $1.1 \mu \mathrm{m} \cdot \mathrm{s}^{-1}$ and $-2.3 \mu \mathrm{m} \cdot \mathrm{s}^{-1}$. As in experiments [Fig. 2(b)], these large triangular oscillations were thus asymmetric, with slower motor movements in the positive direction. Increasing the spring stiffness elicited faster and smaller oscillations in relative amounts similar to those measured (Table I). Near the Hopf bifurcation, the system produced oscillations with magnitudes comparable to $l$ and symmetric waveforms [Fig. 3(c)]. Although this oscillation accounted for most features of the experimental oscillation illustrated in Fig. 2(c), its waveform did not show two welldistinct time scales. This discrepancy may indicate that molecular details of the actomyosin interaction, which are ignored in our theoretical approach, become important at small length scales. Most parameter values obeyed the constraints imposed by experimental estimates. The friction coefficient $\lambda$, however, had to assume very high values, here $\lambda=3-50 \mu \mathrm{N} \cdot \mathrm{s} \cdot \mathrm{m}^{-1}$, compared to that expected from hydrodynamic friction acting on micronsized objects $\left(\sim 0.01 \mu \mathrm{N} \cdot \mathrm{s} \cdot \mathrm{m}^{-1}\right)$. High friction coefficients might emerge from energy losses expected to occur if a motor were to slide along the filament in the bound state before detaching. In support of this inference, a protein friction of $\sim 1 \mu \mathrm{N} \cdot \mathrm{s} \cdot \mathrm{m}^{-1}$ has been recently directly measured with individual kinesin- 8 motor proteins dragged over their microtubule tracks [20].

In conclusion, we showed that an actomyosin system comprising only a single actin filament in interaction with a few tens of myosin molecules can become selfoscillatory when subjected to an elastic load. To our knowledge, this Letter provides the first experimental evidence which suggests that motor molecules working collectively within an assembly can generically power spontaneous oscillations. With a choice of parameter values compatible with experiments, the general description of collective motor oscillations introduced in Ref. [18] could quantitatively reproduce essential features of our experimental observations. Because our motor system is minimal, any complex machinery comprising motor assemblies under load is expected to display the same generic properties. Our findings may thus be relevant for describing mechanical oscillations in a variety of biological systems, including muscles, sensory hair-cell bundles of the inner ear, and, possibly, the mitotic spindle during cell division.

We are indebted to M. Rief for providing the heavy meromyosin molecules. We thank F. Jülicher for discussions, J. Jackson for control experiments, D. Chatenay, A. J. Hudspeth, and E. Schäffer for comments on the manuscript, and B. Lemaire, M. Petit, and the late G. Rousseau from the machine shop of the Institut Curie. This work was supported by Human Frontier Science Program Grant No. RGP0051/2003 and by European Commission FP6 Integrated Project EUROHEAR, LSHG-CT-2004-512063.

*pascal.martin@curie.fr

[1] J. W. Pringle, Proc. R. Soc. B 201, 107 (1978)

[2] S. Ishiwata, Y. Shimamoto, M. Suzuki, and D. Sasaki, Advances in Experimental Medicine and Biology 592, 341 (2007).

[3] A. Fabiato and F. Fabiato, J. Gen. Physiol. 72, 667 (1978).

[4] H. Fujita and S. Ishiwata, Biophys. J. 75, 1439 (1998).

[5] J. Pecreaux et al., Curr. Biol. 16, 2111 (2006).

[6] M. C. Göpfert et al., Proc. Natl. Acad. Sci. U.S.A. 102, 325 (2005).

[7] P. Martin, D. Bozovic, Y. Choe, and A. J. Hudspeth, J. Neurosci. 23, 4533 (2003).

[8] P. Martin and A. J. Hudspeth, Proc. Natl. Acad. Sci. U.S.A. 96, 14306 (1999).

[9] C. Veigel et al., Biophys. J. 75, 1424 (1998).

[10] N. Suzuki, H. Miyata, S. Ishiwata, and K. Kinosita, Biophys. J. 70, 401 (1996).

[11] P. Martin, A.J. Hudspeth, and F. Jülicher, Proc. Natl. Acad. Sci. U.S.A. 98, 14380 (2001).

[12] Y. Shimamoto, F. Kono, M. Suzuki, and S. Ishiwata, Biophys. J. 93, 4330 (2007).

[13] J. Howard, Mechanics of Motor Proteins and the Cytoskeleton (Sinauer Associates, Sunderland, MA, 2001).

[14] A. Vilfan and T. Duke, Biophys. J. 85, 818 (2003).

[15] C. Batters, M.I. Wallace, L.M. Coluccio, and J.E. Molloy, Phil. Trans. R. Soc. B 359, 1895 (2004).

[16] K. E. Machin and J. W. S. Pringle, Proc. R. Soc. B 151, 204 (1959).

[17] D. A. Smith and D. G. Stephenson, Biophys. J. 96, 3682 (2009).

[18] F. Jülicher and J. Prost, Phys. Rev. Lett. 78, 4510 (1997).

[19] B. Nadrowski, P. Martin, and F. Jülicher, Proc. Natl. Acad. Sci. U.S.A. 101, 12195 (2004).

[20] V. Bormuth, V. Varga, J. Howard, and E. Schäffer, Science 325, 870 (2009). 